\title{
sciendo
}

\section{Kinematic Analyses of Parkour Landings From as High as 2.7 Meters}

\author{
by \\ Boyi Dai', Jacob S. Layer ${ }^{1}$, Taylour J. Hinshaw ${ }^{1}$, Ross F. Cook ${ }^{1}$, Janet S. Dufek ${ }^{2}$
}

\begin{abstract}
Developing effective landing strategies has implications for both injury prevention and performance training. The purpose was to quantify the kinematics of Parkour practitioners' landings from three heights utilizing four techniques. Seventeen male and three female Parkour practitioners landed from 0.9, 1.8, and $2.7 \mathrm{~m}$ utilizing the squat, forward, roll, and stiff landing techniques when three-dimensional kinematics were collected. The stiff landing demonstrated the shortest landing time, and the roll landing showed the longest landing time for 1.8 and $2.7 \mathrm{~m}$. Roll landings demonstrated the greatest forward velocities at initial contact and at the end of the landing. Stiff landings showed the greatest changes in vertical velocity during the early landing, while roll landings showed the least changes for 0.9 and $1.8 \mathrm{~m}$. Both roll and stiff landings generally resulted in decreased changes in horizontal velocity during the early landing compared to squat and forward landings. The four landing techniques also demonstrated different lower extremity joint angles. Stiff landings may increase injury risk because of the quick decrease of vertical velocities. Roll landings allow individuals to decrease vertical and horizontal velocities over a longer time, which is likely to decrease the peak loading imposed on the lower extremities.
\end{abstract}

Key words: jump-landing, impact, injury, performance, lower extremities.

\section{Introduction}

During landing tasks, forces and moments are generated by the musculoskeletal system at surface contact to progressively decelerate the velocity of the body (Dufek and Bates, 1990; McNitt-Gray, 1993). When landing after a forward jump, the body's downward velocity must be decelerated by an upward acceleration, while its forward velocity needs to be decelerated by a backward acceleration. Inappropriate landing patterns can cause excessive loading to the body, resulting in musculoskeletal injuries. The anterior cruciate ligament is commonly injured by abnormal landing patterns during athletic activities (Dai et al., 2015b; Krosshaug et al., 2007). Military training also involves jump-landing tasks such as parachuting, jumping off a vehicle, and traversing a ditch; all which increase exposure to jump-landing associated injury risk (Ekeland,
1997; Owens et al., 2007; Sell et al., 2010). Developing safe and effective landing strategies has implications for both injury prevention and performance training.

Investigators have examined the effects of landing heights, distances, and techniques on performers' motion, impact forces, and their associated risk of injury (Dai et al., 2015a; Dufek and Bates, 1990; McNitt-Gray, 1993). Lower extremity loading increases when the landing height and distance are increased (Dufek and Bates, 1990; McNitt-Gray, 1993; Zhang et al., 2000). Potential strategies to decrease lower extremity loading include landing on the forefoot, increasing knee and hip joint range of motion, and lengthening landing time (Dai et al., 2015a; Devita and Skelly, 1992; Zhang et al., 2000). However, previous findings are based on landing heights

1 - Division of Kinesiology and Health, University of Wyoming, Laramie, WY, USA.

2 - Department of Kinesiology and Nutrition Sciences, University of Nevada, Las Vegas, Las Vegas, NV, USA. 
less than $1.5 \mathrm{~m}$ in combination with traditional landing techniques. An increased landing velocity resulting from a high landing height does not necessarily result in injury if appropriate landing techniques are utilized. As an example, Parkour is a form of acrobatic street gymnastics that has gained public popularity in the last decade (Puddle and Maulder, 2013). One important skill in Parkour is to land safely from high heights $(>1.5 \mathrm{~m})$ such as vertical walls. Novel landing techniques with the use of hands and rolling motions have been utilized by Parkour practitioners. Investigators have quantified the effect of Parkour precision and roll landings on landing forces from a landing height of $0.75 \mathrm{~m}$ (Puddle and Maulder, 2013). The biomechanics of how Parkour practitioners land from higher heights remains unclear.

Therefore, the purpose of this study was to quantify the landing kinematics of Parkour practitioners landing from $0.9,1.8$, and $2.7 \mathrm{~m}$ utilizing the squat, forward, roll, and stiff landing techniques. It was hypothesized that the stiff landing would exhibit the least landing time and greatest change in vertical velocity during the early landing, while the roll landing would demonstrate the greatest landing time and least changes in vertical and horizontal velocities during the early landing for all landing heights. In addition, it was hypothesized that the four landing techniques would exhibit different lower extremity kinematics.

\section{Methods}

Participants

Based on a pilot study with two participants and previous studies (Dai et al., 2015a; Dufek and Bates, 1990; Zhang et al., 2000), an effect size of 0.8 was assumed for a paired comparison. A sample size of 15 was needed for a type I error at the level of 0.05 to achieve a power of 0.8. Seventeen male (age: $23.9 \pm 4.7 \mathrm{y}$; body height: $1.78 \pm 5.8 \mathrm{~m}$; body mass: $67.6 \pm 8.5 \mathrm{~kg}$; training experience: $7.1 \pm 2.3 \mathrm{y}$ ) and three female (age: $26.2 \pm 1.3$ y; body height: $1.62 \pm 5.5 \mathrm{~m}$; body mass: $63.0 \pm 13.2 \mathrm{~kg}$; training experience: $4.7 \pm 2.5$ y) Parkour practitioners participated in the study. Participants had a minimum of two-year experience in Parkour training, and practiced Parkour at least twice per week for a total of two hours per week at the time of testing. Individuals were excluded from this study if he or she (1) suffered a major injury which involved surgical treatment within the last five years, (2) suffered a lower extremity injury that prevented participation in physical activity for more than two weeks over the previous six months, (3) possessed any other conditions that prevented him / her from participating at maximal effort in sporting activities, or (4) was pregnant. This study was approved by the University of Wyoming Institute Review Board. Participants signed informed consent forms prior to participation.

Design and Procedures

Data collection was performed in a Parkour training facility. Participants wore their own clothes and shoes. Participants self-reported their maximal landing heights (MLH) in feet. The MLH is the maximal height participants could land from with $100 \%$ confidence and no anxiety. All male participants reported MLH equal or greater than $3.05 \mathrm{~m}$ (10 feet). All female participants reported MLH equal or greater than $2.13 \mathrm{~m}$ (7 feet). To minimize injury risk, participants were asked to land from the following heights as all were equal or below $90 \%$ of their MLH: $0.91 \mathrm{~m}$ (3 feet), $1.83 \mathrm{~m}$ (6 feet), and $2.74 \mathrm{~m}$ (9 feet).

Four landing techniques including the squat, forward, roll, and stiff landings were performed. The squat landing was similar to a traditional soft landing, during which participants landed softly with only feet contacting the ground (Figure 1). For the forward landing, participants landed softly with feet contacting the ground first and leaned their body forward with hands contacting the ground toward the end of the landing (Figure 2). For the roll landing, participants landed softly with a rolling motion from the feet to the hands and to the back (Figure 3). For the stiff landing, participants landed with only feet contacting the ground with a goal to moving from a landing to a forward run as fast as possible (Figure 4). Participants only performed landing techniques they commonly practiced for each landing height. All participants completed four landing techniques from $0.9 \mathrm{~m}$ and $1.8 \mathrm{~m}$. All male participants completed the squat, forward, and roll landings from $2.7 \mathrm{~m}$.

Participants were given unlimited time to perform a self-selected warm-up. Additionally, unlimited practice trials were permitted before all 
jump height/technique combinations. However, most participants only performed one practice trial for each combination. Participants performed one recorded landing trial for each landing technique from each landing height. All participants initiated the takeoff in a deep squat position. Four starting locations were utilized for different landing conditions (Figures 5). The starting location for the $2.7 \mathrm{~m}$ roll landing was behind the other starting positions because a roll landing from that height was typically initiated with greater forward velocities. To minimize injury risk, jump heights progressed from lowest to highest; however, the landing technique order was randomized for each participant. Between each landing, participants rested for a minimum of $30 \mathrm{~s}$. No injuries occurred during data collection.

For motion capture, three JVC GC-PX10 camcorders (JVC, Tokyo, Japan) were used with a resolution of 1920 by 1080 pixels, a frame rate of 59.94 frames / s, and a shutter speed of $1 / 400 \mathrm{~s}$. The three camcorders were placed to capture the front, right, and left views of landings. A 32marker calibration frame (Peak Performance; Englewood, CO, USA) covering a volume of $2.5 \times$ $2 \times 2 \mathrm{~m}$ was used for calibration. Two landing areas were calibrated for different landing heights and techniques (Figure 5). A global reference frame was established using markers placed on the ground with forward, left, and upward directions designated as positive.

Data Reduction

Calibrations were performed between the frontal and right camcorders and between the frontal and left camcorders using the direct linear transformation procedure (Abdel-Aziz and Karara, 1971). The calibration error was $5 \pm 2 \mathrm{~mm}$ for different calibration points in the current study. For videos captured by the front camcorder, twenty-one body landmarks, including vertex (top of the head) and bilateral gonions, shoulders (center of the humeral head), elbows (mid-point between medial and lateral epicondyles), wrists (mid-point between radial and ulnar styloid processes), third metacarpal heads, greater trochanters, knees (mid-point between medial and lateral femoral condyles), ankles (mid-point between medial and lateral malleoli), tips of toes, and bottoms of heels (Hay, 1993), were manually digitized (Figures 1-4). For videos captured by the right camcorder, the body landmarks on the right side and vertex were digitized. For videos captured by the left camcorder, the body landmarks on the left side were digitized. Body landmarks were digitized every frame starting four frames before initial ground contact to at least four frames after obvious upward movements were visible or either foot left the ground. Three camcorders were synchronized using the first foot's initial ground contact. Videos were edited using Adobe Premiere Pro CS5.5 software (Adobe Systems, Inc, San Jose, CA, U.S.). Manual digitization was performed using MaxTRAQ software (Innovision Systems, Inc, Columbiaville, MI, U.S.).

Three-dimensional coordinates of body landmarks were obtained from the synchronized two-dimensional coordinates and calibration parameters of each camcorder (Abdel-Aziz and Karara, 1971; Dai et al., 2015b). Three-dimensional coordinates were then filtered using a Butterworth fourth-order zero-lag low-pass filter with a cut-off frequency of $7.14 \mathrm{~Hz}$. This cut-off frequency was based on Equation 1 ( $\mathrm{Yu}$ and Andrews, 1998), which was derived from simulated data with random noise and estimated optimal cut-off frequencies from sampling frequencies. Previous studies have used this method for jump-landing, javelin throwing, and discus throwing (Beardt et al., 2018; Dai et al., 2015b; Leigh et al., 2008). Hip centers were defined as $25 \%$ of the distance from the ipsilateral to the contralateral greater trochanters (Bennett et al., 2016), and other joint centers were directly digitized. The mass and center of mass (COM) positions of individual segments were determined based on the literature (de Leva, 1996). Positions of the whole-body COM were calculated using the segmentation method (Hay, 1993). Velocities of the whole-body COM were calculated as the changes in positions over time using the finite difference method.

$F_{c}=\left(1.4845+0.1532 \sqrt{F_{s}}\right)^{2} \quad$ Equation 1, where: $\mathrm{F}_{\mathrm{c}}=$ cut-off frequency; $\mathrm{F}_{\mathrm{s}}=$ sampling frequency

The end of the landing was defined as the first frame where either vertical velocities of the whole-body COM became positive or either foot left the ground, whichever occurred earlier. Landing time, vertical and horizontal velocities of 
the whole-body COM at initial contact and at the end of the landing, as well as changes in vertical and horizontal velocities of the whole-body COM during the first $100 \mathrm{~ms}$ of landing were extracted as whole-body kinematics for analysis. The first $100 \mathrm{~ms}$ of the landing were chosen as peak impact ground reaction forces (GRFs) occurred in the first $100 \mathrm{~ms}$ of the landing and could occur at as early as $20 \mathrm{~ms}$ of the landing (Dai et al., 2015a). In addition, anterior cruciate ligament injuries were found to occur during this time window (Koga et al., 2010). The trunk was defined as a vector passing from the mid-hip to the mid-shoulder while thighs, shanks, and feet were defined as vectors passing from the distal joints to the proximal joints. Joint angles were calculated as the angle between two adjacent segments. Ankle, knee, and hip joint angles at initial contact as well as peak ankle dorsiflexion, peak knee and hip flexion angles for the right leg were extracted for analysis, as the left and right legs demonstrated similar kinematics. Calculations were performed using customized subroutines developed in MATLAB 2016b (MathWorks Inc. Natick, MA).

Statistical Analysis

Because females did not perform any landings from $2.7 \mathrm{~m}$ and males did not perform stiff landings from $2.7 \mathrm{~m}$, a complete three by four repeated measure analysis of variance (ANOVA) could not be performed. Instead, variables were compared among the three landing heights for each landing technique or among the four landing techniques for each landing height using multiple one-way repeated measure ANOVAs. When oneway ANOVAs showed significant main effects, paired $\mathrm{t}$-tests were performed between each pair of two landing conditions. A type-I error rate was set at 0.05 for ANOVAs for statistical significance. The Benjamini-Hochberg procedure was applied to all paired t-tests to control the study-wide false discovery rate to be 0.05 (Benjamini and Hochberg, 1995). To perform this procedure, the $p$ values of all paired $\mathrm{t}$-tests were ranked from the smallest to the greatest. The $p$ value of each test was then compared to a critical value of $0.05^{*} \mathrm{i} / \mathrm{n}$ for statistical significance, where $i$ was the individual $p$-value's rank and $\mathrm{n}$ was the total number of tests. Statistical analyses were performed using the SPSS Statistics 24 software (IBM Corporation, Armonk, NY, USA).

\section{Results}

Significant main effects were observed for most one-way ANOVAs. The largest $p$ value for a significant paired t-test was 0.038 after the adjustment for the false discovery rate. Vertical and horizontal velocities from initial contact to the end of the landing for all landing conditions were provided for one participant (Figure 6). For whole-body kinematics (Table 1), as the landing height increased, landing time decreased for the squat, forward, and roll landings, but increased for the stiff landing. The stiff landing demonstrated the shortest landing time for $0.9 \mathrm{~m}$ and $1.8 \mathrm{~m}$, and the roll landing showed the longest landing time for $1.8 \mathrm{~m}$ and $2.7 \mathrm{~m}$. Downward velocities at initial contact increased with landing height for all landing techniques, with the stiff landing showing lower downward velocities for $0.9 \mathrm{~m}$ and $1.8 \mathrm{~m}$ compared to the other three landings. The magnitudes of vertical velocities at the end of the landing were generally similar (0.1-0.2 m/s) across all landing heights for the squat, forward, and stiff landings. The roll landings resulted in downward velocities at the end of the landing compared to upward velocities in most other conditions. Horizontal velocities at initial contact did not significantly change as landing height increased for the squat, forward, and stiff landings, but those velocities increased from $0.9 \mathrm{~m}$ and $1.8 \mathrm{~m}$ to $2.7 \mathrm{~m}$ for the roll landing, which also demonstrated the greatest forward velocities at initial contact among four landing techniques. Similarly, horizontal velocities at the end of the landing did not significantly change as landing height increased for the squat and forward landings, but they increased as landing height increased for the roll landing. Both the roll and stiff landings also showed greater forward velocities at the end of the landing than the squat and forward landings. Changes in vertical velocity during the first $100 \mathrm{~ms}$ of landing increased as the landing height increased for all landing techniques. Changes in vertical velocity were the greatest for the stiff landing, the second greatest for the squat landing, the third greatest for the forward landing, and the least for the roll landing for 0.9 and $1.8 \mathrm{~m}$. Changes in horizontal velocities during the first $100 \mathrm{~ms}$ of the landing were generally similar across landing heights for the squat, forward, and stiff landings. 
Table 1

Whole-body kinematics for three landing heights and four landing techniques and $p$ values of ANOVAs

\begin{tabular}{|c|c|c|c|c|c|c|}
\hline \multirow[t]{2}{*}{ Variables } & \multirow[t]{2}{*}{ Landing Heights (m) } & \multicolumn{4}{|c|}{ Landing Techniques } & \multirow{2}{*}{$\begin{array}{l}p \text { values, } \\
\text { Landing } \\
\text { Technique }\end{array}$} \\
\hline & & Squat & Forward & Roll & Stiff & \\
\hline \multirow{7}{*}{ Landing Time (ms) } & \multirow{2}{*}{0.9} & $377.0 \pm 74.5$ & $381.2 \pm 46.6$ & $379.5 \pm 59.5$ & $168.5 \pm 51.6$ & \multirow{2}{*}{$<0.001$} \\
\hline & & A, I & A, I & $\mathrm{A}, \mathrm{I}$ & B, II & \\
\hline & \multirow{2}{*}{1.8} & $334.5 \pm 51.8$ & $321.2 \pm 39.4$ & $363.7 \pm 65.5$ & $223.6 \pm 43.1$ & \multirow{2}{*}{$<0.001$} \\
\hline & & B, I-II & B, II & $\mathrm{A}, \mathrm{I}$ & A, III & \\
\hline & \multirow{2}{*}{2.7} & $289.5 \pm 29.5$ & $288.5 \pm 23.4$ & $319.9 \pm 36.5$ & & \multirow{2}{*}{$<0.001$} \\
\hline & & C, II & C, II & $\mathrm{B}, \mathrm{I}$ & --- & \\
\hline & $p$ values, Landing Height & $<0.001$ & $<0.001$ & 0.001 & $<0.001$ & \\
\hline \multirow{6}{*}{$\begin{array}{l}\text { Vertical Velocity at } \\
\text { Initial Contact } \\
(\mathrm{m} / \mathrm{s})\end{array}$} & \multirow{2}{*}{0.9} & $-3.0 \pm 0.2$ & $-3.1 \pm 0.3$ & $-3.0 \pm 0.2$ & $-2.8 \pm 0.3$ & \multirow{2}{*}{$<0.001$} \\
\hline & & C, I & C, I & C, I & B, II & \\
\hline & \multirow{2}{*}{1.8} & $-4.9 \pm 0.1$ & $-4.9 \pm 0.1$ & $-4.8 \pm 0.2$ & $-4.8 \pm 0.1$ & \multirow{2}{*}{0.003} \\
\hline & & B, I & $\mathrm{B}, \mathrm{I}$ & B, I & A, II & \\
\hline & \multirow{2}{*}{2.7} & $-6.3 \pm 0.1$ & $-6.3 \pm 0.1$ & $-6.3 \pm 0.1$ & & \multirow{2}{*}{0.08} \\
\hline & & A, I & A, I & A, I & -- & \\
\hline \multirow{8}{*}{$\begin{array}{l}\text { Vertical Velocity at } \\
\text { the End of Landing } \\
(\mathrm{m} / \mathrm{s})\end{array}$} & $p$ values, Landing Height & $<0.001$ & $<0.001$ & $<0.001$ & $<0.001$ & \multirow{3}{*}{0.004} \\
\hline & & $0.0 \pm 0.0$ & $0.1 \pm 0.1$ & $-0.2 \pm 0.3$ & $-0.2 \pm 0.4$ & \\
\hline & 0.9 & C, II & $\mathrm{B}, \mathrm{I}$ & A, III & B, III & \\
\hline & 18 & $0.1 \pm 0.0$ & $0.1 \pm 0.1$ & $-0.4 \pm 0.2$ & $0.1 \pm 0.1$ & $<0,001$ \\
\hline & 1.0 & B, I & A-B, I & A, II & A, I & $<0.001$ \\
\hline & & $0.1 \pm 0.1$ & $0.2 \pm 0.1$ & $-0.3 \pm 0.3$ & & \\
\hline & 2.7 & A, I & A, I & A, II & --- & $<0.001$ \\
\hline & $p$ values, Landing Height & 0.001 & 0.02 & 0.10 & 0.001 & \\
\hline & 0.9 & $1.4 \pm 0.3$ & $1.5 \pm 0.3$ & $1.8 \pm 0.4$ & $1.7 \pm 0.5$ & \\
\hline & & A, II & A, II & B, I & A, I-II & 0.001 \\
\hline Horizontal & 18 & $1.4 \pm 0.2$ & $1.5 \pm 0.3$ & $1.9 \pm 0.4$ & $1.6 \pm 0.3$ & \\
\hline Velocity at Initial & 1.8 & A, II & A, II & $\mathrm{B}, \mathrm{I}$ & A, II & $<0.001$ \\
\hline Contact (m/s) & & $1.4 \pm 0.3$ & $1.4 \pm 0.2$ & $2.6 \pm 0.3$ & & \\
\hline & 2.7 & A, II & A, II & A, I & --- & $<0.001$ \\
\hline & $p$ values, Landing Height & 0.65 & 0.11 & $<0.001$ & 0.4 & \\
\hline & & $0.2 \pm 0.1$ & $0.5 \pm 0.3$ & $2.3 \pm 0.4$ & $2.1 \pm 0.5$ & $<0.001$ \\
\hline & 0.9 & A, III & A, II & C, I & A, I & $<0.001$ \\
\hline Horizontal & & $0.2 \pm 0.2$ & $0.5 \pm 0.2$ & $2.5 \pm 0.5$ & $1.8 \pm 0.6$ & \\
\hline Velocity at the End & 1.8 & A, IV & A, III & B, I & B, II & $<0.001$ \\
\hline of Landing (m/s) & 27 & $0.2 \pm 0.2$ & $0.5 \pm 0.6$ & $2.8 \pm 0.4$ & & \\
\hline & 2.7 & A, III & A, II & A, I & --- & $<0.001$ \\
\hline & $p$ values, Landing Height & 0.86 & 0.78 & $<0.001$ & 0.03 & \\
\hline & 0.9 & $1.1 \pm 0.4$ & $0.8 \pm 0.4$ & $0.5 \pm 0.4$ & $1.4 \pm 0.5$ & $<0,001$ \\
\hline Change in Vertical & 0.9 & C, II & C, III & C, IV & $\mathrm{B}, \mathrm{I}$ & $<0.001$ \\
\hline Velocity during the & & $2.4 \pm 0.3$ & $2.2 \pm 0.2$ & $2.0 \pm 0.3$ & $2.8 \pm 0.5$ & \\
\hline First $100 \mathrm{~ms}$ of & 1.8 & B, II & B, III & B, IV & A, I & $<0.001$ \\
\hline landing $(\mathrm{m} / \mathrm{s})$ & 27 & $3.2 \pm 0.3$ & $3.3 \pm 0.2$ & $3.2 \pm 0.3$ & & \\
\hline & 2.7 & A, I & A, I & A, I & --- & 0.88 \\
\hline & $p$ values, Landing Height & $<0.001$ & $<0.001$ & $<0.001$ & $<0.001$ & \\
\hline & 09 & $-0.5 \pm 0.2$ & $-0.5 \pm 0.2$ & $-0.2 \pm 0.2$ & $0.1 \pm 0.3$ & \\
\hline $\begin{array}{l}\text { Change in } \\
\text { Horizontal }\end{array}$ & 0.9 & A, I & A, I & A, II & B, III & $<0.001$ \\
\hline Velocity during the & 1.8 & $-0.6 \pm 0.2$ & $-0.5 \pm 0.2$ & $-0.1 \pm 0.2$ & $-0.1 \pm 0.4$ & \\
\hline First $100 \mathrm{~ms}$ of & 1.0 & A, I & A, I & B, II & A, II & $<0.001$ \\
\hline landing $(\mathrm{m} / \mathrm{s})$ & & $-0.6 \pm 0.2$ & $-0.4 \pm 0.2$ & $-0.3 \pm 0.2$ & & \\
\hline landing (m/s) & 2.7 & A, I & A, II & A, II & --- & 0.01 \\
\hline & $p$ values, Landing Height & 0.59 & 0.07 & 0.002 & 0.03 & \\
\hline
\end{tabular}

Notes: Upward and forward directions were designated as positive, while downward and backward directions were designated as negative. The effect of landing technique for each landing height was grouped, where I $>I I>I I I>I V$. The effect of landing height for each landing technique was grouped, where $A>B>C$. Conditions with more than one symbol indicated non-significant differences compared to other conditions with one of the same symbols. For example, $A-B$ indicated non-significant differences compared to conditions with $A$ or $B$. Indicated group differences were significant at $p<0.05$ false discovery rate-adjusted Type I error. ---: Data not collected for this condition. 
Table 2

Lower extremity joint kinematics for three landing heights and four landing techniques and $p$ values of ANOVAs

\begin{tabular}{|c|c|c|c|c|c|c|}
\hline \multirow[t]{2}{*}{ Variables } & \multirow[t]{2}{*}{ Landing Heights (m) } & \multicolumn{4}{|c|}{ Landing Techniques } & \multirow{2}{*}{$\begin{array}{c}p \text { values, } \\
\text { Landing } \\
\text { Technique }\end{array}$} \\
\hline & & Squat & Forward & Roll & Stiff & \\
\hline \multirow{7}{*}{$\begin{array}{l}\text { Ankle } \\
\text { Plantarflexion at } \\
\text { Initial Contact }\left({ }^{\circ}\right)\end{array}$} & \multirow{2}{*}{0.9} & $26.1 \pm 6.4$ & $27.1 \pm 7.1$ & $15.4 \pm 8.7$ & $15.6 \pm 11.8$ & \multirow{2}{*}{$<0.001$} \\
\hline & & A, I & $\mathrm{A}, \mathrm{I}$ & B, II & B, II & \\
\hline & \multirow{2}{*}{1.8} & $28.1 \pm 6.1$ & $27.0 \pm 6.0$ & $22.9 \pm 7.4$ & $25.0 \pm 5.9$ & \multirow{2}{*}{$<0.001$} \\
\hline & & A, I & A, I-II & A, III & A, II-III & \\
\hline & \multirow{2}{*}{2.7} & $27.0 \pm 5.2$ & $25.9 \pm 6.8$ & $22.2 \pm 6.1$ & & \multirow{3}{*}{$<0.001$} \\
\hline & & A, I & A, I & A, II & --- & \\
\hline & $p$ values, Landing Height & 0.09 & 0.77 & $<0.001$ & 0.001 & \\
\hline \multirow{7}{*}{$\begin{array}{l}\text { Peak Ankle } \\
\text { Dorsiflexion }\left({ }^{\circ}\right)\end{array}$} & 09 & $-22.9 \pm 5.0$ & $-26.2 \pm 7.1$ & $-36.1 \pm 8.1$ & $-33.6 \pm 9.0$ & \multirow{2}{*}{$<0.001$} \\
\hline & 0.9 & A, III & A, II & A-B, I & A, I & \\
\hline & \multirow{2}{*}{1.8} & $-24.2 \pm 6.3$ & $-28.3 \pm 7.6$ & $-37.1 \pm 8.8$ & $-33.8 \pm 9.1$ & \multirow{2}{*}{$<0.001$} \\
\hline & & A, IV & A, III & A, I & A, II & \\
\hline & \multirow{2}{*}{2.7} & $-23.5 \pm 6.7$ & $-29.5 \pm 8.1$ & $-33.3 \pm 10.0$ & & \multirow{3}{*}{$<0.001$} \\
\hline & & A, III & A, II & $\mathrm{B}, \mathrm{I}$ & --- & \\
\hline & $p$ values, Landing Height & 0.63 & 0.10 & 0.012 & 0.92 & \\
\hline \multirow{7}{*}{$\begin{array}{l}\text { Knee Flexion at } \\
\text { Initial Contact }\left({ }^{\circ}\right)\end{array}$} & \multirow{2}{*}{0.9} & $21.0 \pm 6.7$ & $23.9 \pm 6.6$ & $28.7 \pm 9.9$ & $21.9 \pm 10.3$ & \multirow{2}{*}{0.008} \\
\hline & & A, II & A, II & A, I & A, II & \\
\hline & & $20.7 \pm 3.6$ & $21.2 \pm 4.2$ & $20.3 \pm 6.4$ & $18.5 \pm 4.6$ & \\
\hline & 1.8 & A, I & A, I & B, I & A, I & 0.23 \\
\hline & & $21.3 \pm 3.0$ & $20.2 \pm 4.7$ & $19.6 \pm 5.4$ & & \\
\hline & 2.7 & A, I & A, I & B, I & --- & 0.36 \\
\hline & $p$ values, Landing Height & 0.88 & 0.10 & $<0.001$ & 0.14 & \\
\hline & 0.9 & $\begin{array}{c}115.6 \pm 14.4 \\
\text { C, II }\end{array}$ & $\begin{array}{l}135.1 \pm 8.3 \\
\quad \text { A, I }\end{array}$ & $\begin{array}{c}120.1 \pm 14.8 \\
\text { A, II }\end{array}$ & $\begin{array}{c}77.0 \pm 11.5 \\
\text { B, III }\end{array}$ & $<0.001$ \\
\hline & & $125.8 \pm 11.0$ & $134.9 \pm 9.4$ & $115.4 \pm 15.7$ & $94.1 \pm 14.9$ & \\
\hline Peak Knee Flexion & 1.8 & B, II & A, I & A, III & A, IV & $<0.001$ \\
\hline (") & & $134.4 \pm 10.7$ & $139.6 \pm 10.2$ & $121.3 \pm 16.3$ & & \\
\hline & 2.7 & A, II & A, I & A, III & --- & 0.001 \\
\hline & $p$ values, Landing Height & $<0.001$ & 0.03 & 0.25 & $<0.001$ & \\
\hline & 00 & $46.2 \pm 12.5$ & $55.8 \pm 12.1$ & $67.0 \pm 11.8$ & $41.9 \pm 13.8$ & \\
\hline & 0.9 & A, III & A, II & A, I & A, III & $<0.001$ \\
\hline & 18 & $34.7 \pm 7.5$ & $40.0 \pm 11.4$ & $41.5 \pm 9.1$ & $33.6 \pm 9.5$ & 0001 \\
\hline Inp Fitial Contact $\left(^{\circ}\right)$ & 1.8 & B, III & B, I-II & B, I & B, II-III & 0.001 \\
\hline & & $31.6 \pm 5.6$ & $34.7 \pm 7.7$ & $36.3 \pm 5.9$ & & \\
\hline & 2.7 & B, I & C, I & B, I & --- & 0.06 \\
\hline & $p$ values, Landing Height & $<0.001$ & $<0.001$ & $<0.001$ & 0.003 & \\
\hline & & $132.0 \pm 13.4$ & $144.5 \pm 7.0$ & $136.6 \pm 11.6$ & $61.2 \pm 16.0$ & \\
\hline & 0.9 & C, II & A, I & A, II & B, III & $<0.001$ \\
\hline & 18 & $147.4 \pm 7.5$ & $141.8 \pm 6.0$ & $130.5 \pm 11.4$ & $98.1 \pm 23.6$ & 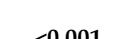 \\
\hline Peak Hip Flexion & 1.8 & B, I & A, II & B, III & A, IV & $<0.001$ \\
\hline & 27 & $152.1 \pm 5.0$ & $143.6 \pm 7.3$ & $135.4 \pm 9.8$ & & $\leq 0001$ \\
\hline & 2.7 & A, I & A, II & A-B, III & -- & $<0.001$ \\
\hline & $p$ values, Landing Height & $<0.001$ & 0.23 & 0.01 & $<0.001$ & \\
\hline
\end{tabular}

Notes: The effect of landing technique for each landing height was grouped, where I $>I I>$ $I I I>I V$. The effect of landing height for each landing technique was grouped, where $A>B$ $>C$. Conditions with more than one symbol indicated non-significant differences compared to other conditions with one or more of the same symbols. For example, A-B indicated nonsignificant differences compared to conditions with $A$ or $B$. Indicated group differences were significant at $p<0.05$ false discovery rate-adjusted Type I error. ---: Data not collected for this condition. 


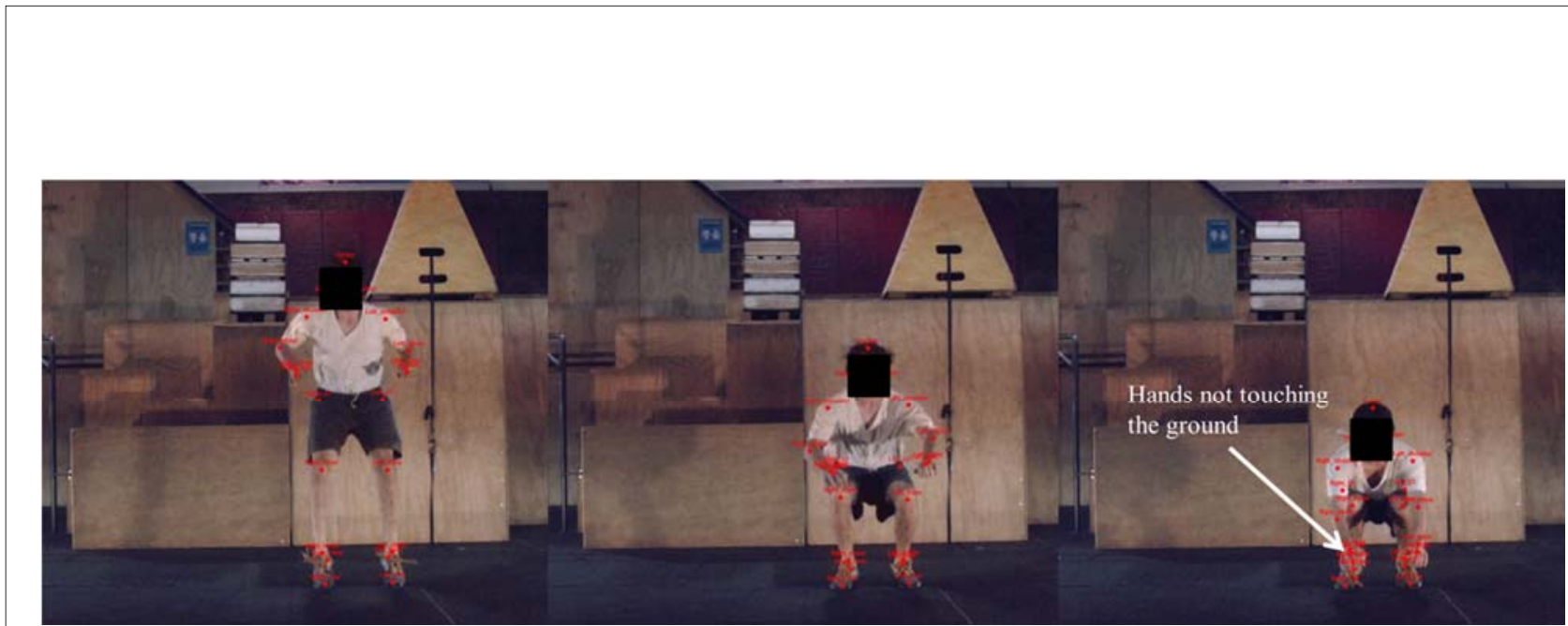

Figure 1

Squat landing

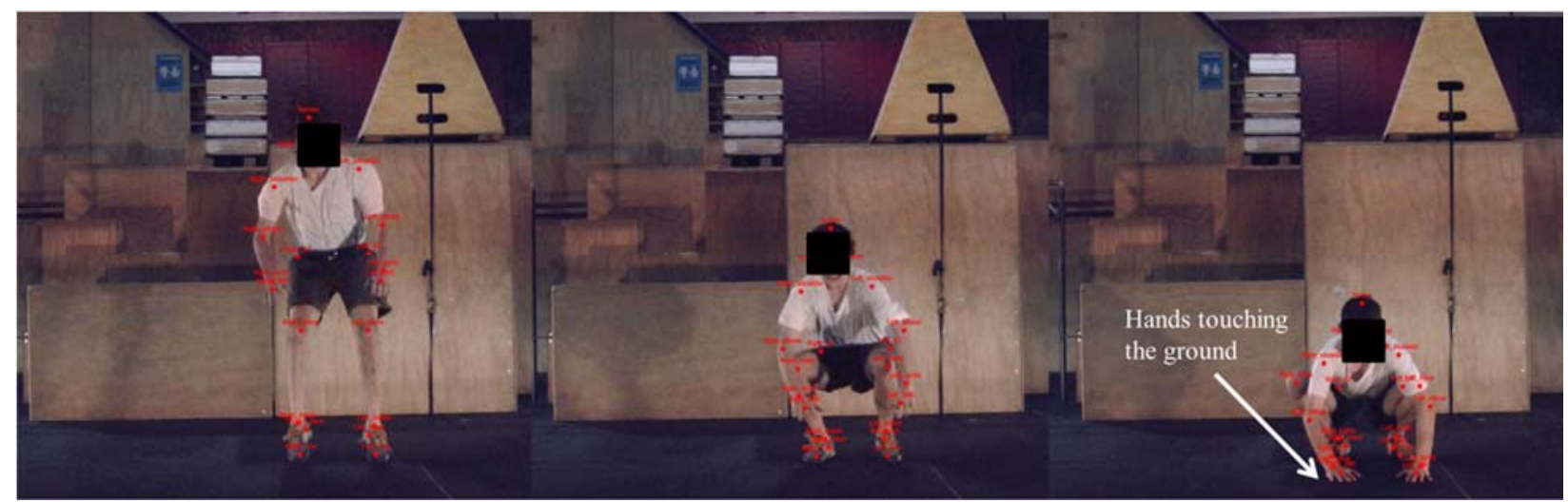

Figure 2

Forward landing

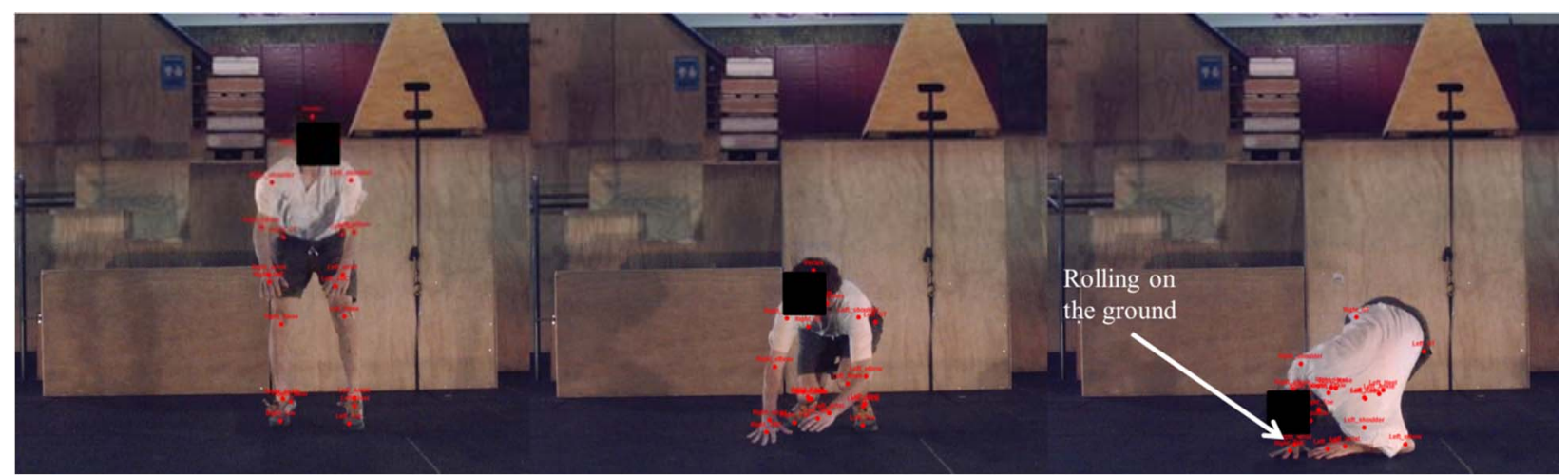

Figure 3

Roll landing 


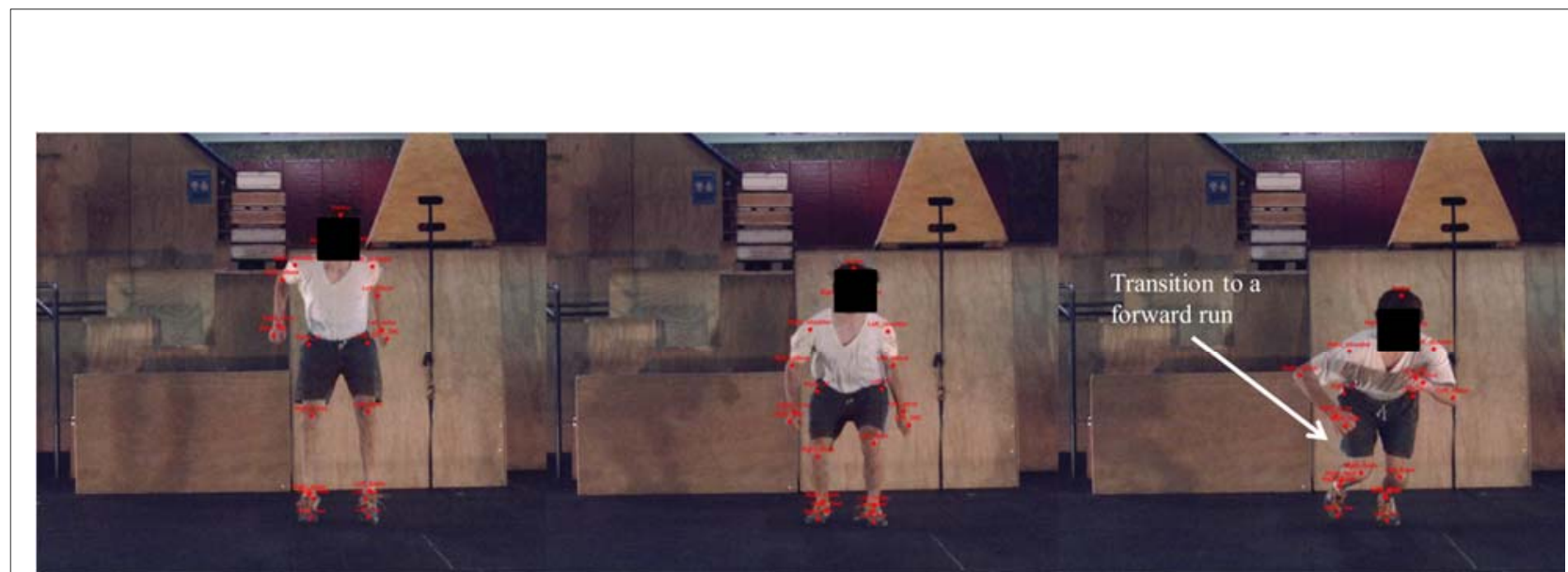

Figure 4

Stiff landing

\begin{tabular}{c}
$0 .-1$ \\
\hdashline$\ldots . .$.
\end{tabular}

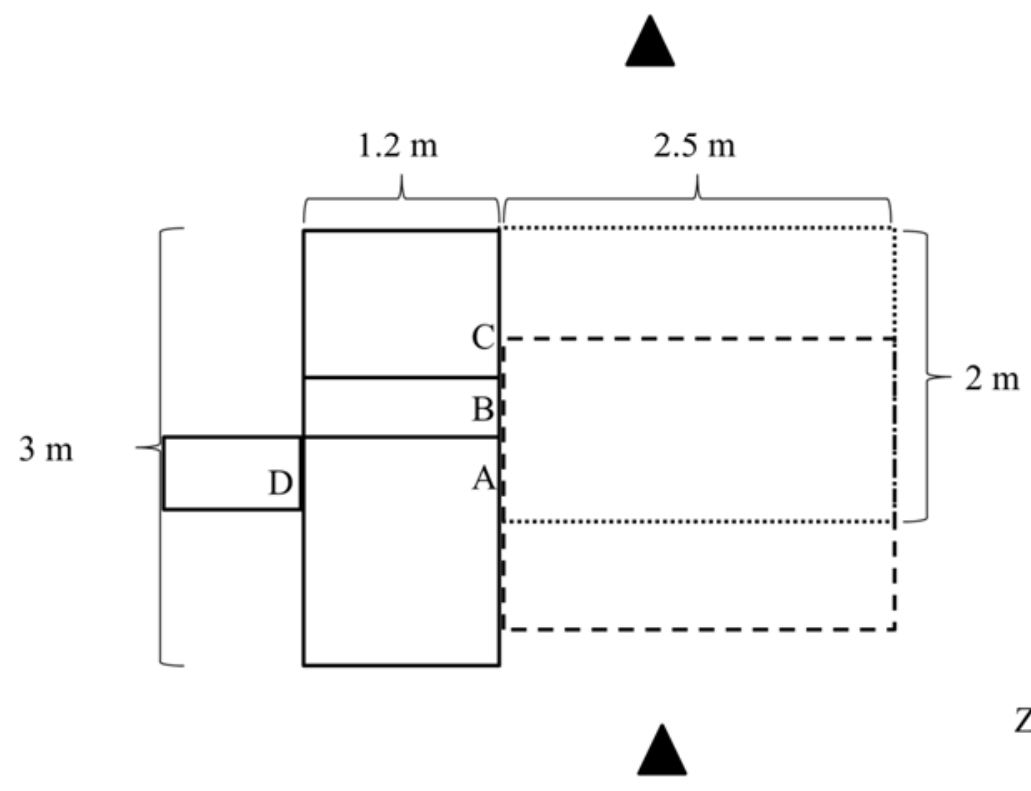

A: Takeoff location for landings from $0.9 \mathrm{~m}$

B: Takeoff location for landings from $1.8 \mathrm{~m}$

C: Takeoff location for squat and forward landings from $2.7 \mathrm{~m}$

D: Takeoff location for roll landings from $2.7 \mathrm{~m}$

Figure 5

Set-up for data collection 

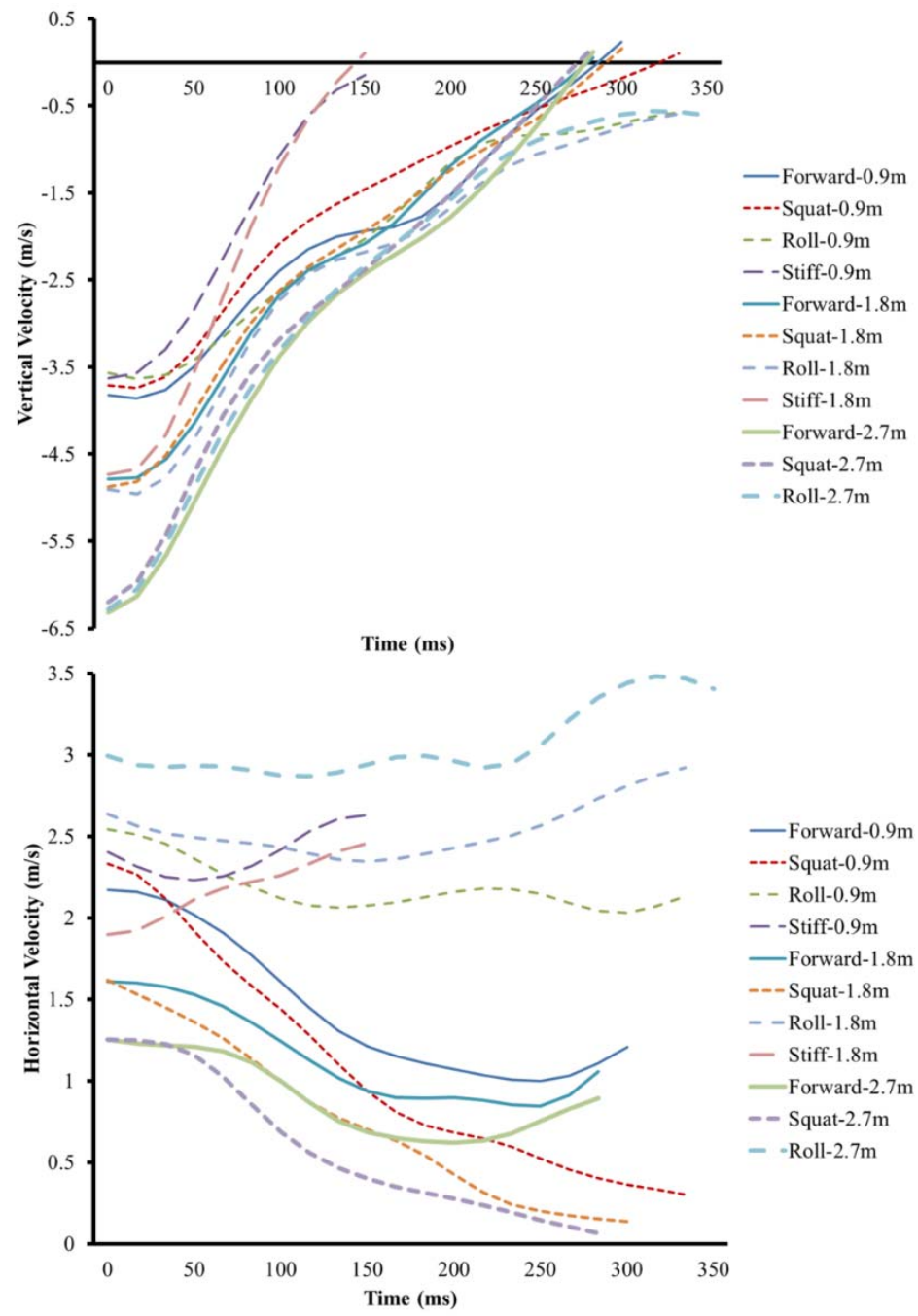

Figure 6

Vertical (top) and horizontal (bottom) velocities from initial contact to the end of the landing for all landing conditions for one participant.

Roll and stiff landings generally resulted in decreased changes in horizontal velocities compared to squat and forward landings.

Regarding joint kinematics (Table 2), roll and stiff landings displayed increased ankle plantar flexion angles at initial contact when landing height increased from 0.9 to $1.8 \mathrm{~m}$, but these angles were lower than those seen during the squat and forward techniques. Peak ankle dorsiflexion angles did not significantly change as landing height increased for the squat, forward, and stiff landings, but those angles were the greatest for roll and stiff landings and the least for the squat landing. Knee flexion angles at initial 
contact did not significantly change as landing height increased for the squat, forward, and stiff landings, but those angles were similar for 1.8 and $2.7 \mathrm{~m}$, but greater for $0.9 \mathrm{~m}$ for the roll landing. Peak knee flexion angles increased as the landing height increased for the squat and stiff landings. Peak knee flexion angles were generally the greatest for the forward landing and the least for the stiff landing for all landing heights. Hip flexion angles at initial contact decreased with increased landing height for all landing techniques and were generally greatest for the roll landing and least for the stiff landing. Peak hip flexion angles increased as the landing height increased for the squat and stiff landings, and were greatest for the squat landing and the least for the stiff landing for 1.8 and $2.7 \mathrm{~m}$.

\section{Discussion}

The findings support the hypothesis that the stiff landing would demonstrate the least landing time and greatest change in vertical velocity during the early landing for 0.9 and 1.8 $\mathrm{m}$. Stiff landings are commonly performed by Parkour practitioners when attempting to transition from a landing to a forward run as fast as possible during competition. The stiff landing was characterized by the least knee and hip flexion angles at initial contact, resulting in earlier contact with ground and slightly decreased downward velocity at initial contact. After initial contact, participants limited knee and hip joint range of motion to decrease landing time. Consequently, a greater change of vertical velocity was observed during the early landing. In addition, landing time increased from 0.9 to $1.8 \mathrm{~m}$ for the stiff landing, indicating participants needed longer time to decelerate the increased downward velocities at initial contact before moving to a forward run. On the other hand, landing time decreased as landing height increased for the other three landing techniques, suggesting the increased downward velocities at initial contact limited participants' ability to decelerate these velocities as slowly as possible. The different performance goals of the stiff landing and other three landings resulted in opposite changes in landing time as a function of landing height. In the horizontal direction, forward velocities were maintained from initial contact to the end of the landing for the stiff landing, consistent with decreased changes of horizontal velocity during the early landing. Stiff landings have been shown to result in increased impact GRFs (Dai et al., 2019; Devita and Skelly, 1992; Zhang et al., 2000), which may increase the load placed on the lower extremities (Dai et al., 2014). In the current study, landing height was considerably greater than in previous studies (Dufek and Bates, 1990; McNitt-Gray, 1993; Zhang et al., 2000), but GRFs were not assessed. Acceleration data, which were directly related to forces, were not reported because of noise introduced by numerical differentiation. Instead, changes in velocities during the early landing were calculated to understand the average force applied to the participant based on the impulsemomentum theorem. The increased change of vertical velocity resulting from the stiff landing was associated with increased average vertical GRFs, which were consistent with previously observed increased impact GRFs (Dai et al., 2019; Devita and Skelly, 1992; Zhang et al., 2000), indicating potential increases in lower extremity loading and injury risk (Dai et al., 2014).

The hypothesis that the roll landing would demonstrate the longest landing time and least changes in vertical and horizontal velocities was generally supported. Participants increased forward lean of the trunk for the roll landing, as shown by the increased hip flexion at initial contact. The forward lean of the trunk also moved the shank segment forward, resulting in increased ankle dorsiflexion. Toward the end of the roll landing, the whole-body rolled forward as support was transitioned from the feet to the hands and then to the back, as opposed to the further increases in knee and hip joint angles characterizing the squat and forward landings. Consequently, the roll landing generally demonstrated decreased peak knee and hip joint flexion compared to the squat and forward landings. The whole-body rolling motion allowed participants to further lower their body to increase landing time, which may have contributed to the decreased changes in vertical velocity during the early landing for 0.9 and 1.8 $\mathrm{m}$. However, changes in vertical velocities for 2.7 $\mathrm{m}$ were similar among the squat, forward, and roll landings, suggesting a limited effect of the rolling motion to gradually decrease vertical velocities for the greatest height. Puddle and Maulder (2013) quantified vertical GRFs and 
loading rates when male Parkour practitioners landed from $0.75 \mathrm{~m}$ using a traditional landing, a precision landing, and a roll landing. The researchers found that both the precision and roll landings resulted in decreased peak vertical GRFs and loading rates versus the traditional landing. Direct comparisons between this previous study and the current study may be difficult due to different landing heights, techniques, and biomechanical measurements reported. However, both studies support that the roll landing may decrease vertical loading from certain heights. When the squat and forward landings were compared, the utilization of hands at the end of the landing decreased changes in vertical velocity during the early landing for the forward landing. At $2.7 \mathrm{~m}$, the forward landing showed the greatest peak knee flexion while the squat landing showed the greatest hip flexion. This may have resulted from a more anterior COM position for the forward landing compared to the squat landing.

The most distinct differences among the squat, forward, and roll landings were in the horizontal direction. The roll landing started and ended with the greatest forward velocities, but also resulted in the least changes in horizontal velocity during the early landing. While forward velocities were decelerated toward zero for the squat and forward landings, they were mostly maintained throughout the landing for the roll landing. Dufek and Bates (1990) assessed impact vertical GRFs during landing tasks with a variety of landing heights, distances, and techniques. Both the first and second maximum vertical GRFs increased as the horizontal distance increased. Cruz et al. (2013) compared landing biomechanics for drop-landings and a forward jump-landing. The forward jump imposed a greater forward velocity at initial contact, and subsequently resulted in increased peak anterior tibial shear forces and knee joint moments, compared to landings without a forward velocity component. Therefore, when an initial forward velocity needs to be decelerated in addition to an initial downward velocity, lower extremity loading is likely to increase due to increased vertical and posterior GRFs and joint moments (Dai et al., 2014; Lin et al., 2009). In the current study, although participants were not asked to jump over a horizontal distance, they preferred to initiate the takeoff with a forward velocity for the roll landings. When the downward velocity was largely attenuated during the vertical landing phase, the forward velocity could be subsequently transferred to a run or decreased over a horizontal distance. The roll landing will be particularly effective when individuals have to jump over a horizontal distance from the takeoff to the landing location, imposing greater forward velocity at initial contact.

The current findings may provide information for developing landing training strategies for Parkour practitioners, athletes, and military personnel. First, by starting with a deep squat position and contacting the ground with a slightly flexed posture, the effective landing height decreased and resulted in decreased downward velocity at initial contact compared to theoretical values $(4.2 \mathrm{~m} / \mathrm{s}$ for $0.9 \mathrm{~m}, 6.0 \mathrm{~m} / \mathrm{s}$ for 1.8 $\mathrm{m}$, and $7.3 \mathrm{~m}$ for $2.7 \mathrm{~m}$ ). Second, stiff landings may increase injury risk because of the greatest change in vertical velocity during the early landing and should only be performed from certain heights with sufficient practice and preparation. Third, if the goal is to complete a vertical landing without an initial forward velocity or a secondary task after the landing, the roll landing may provide some advantages in decreasing changes in vertical velocity for two lower heights, but not for the greatest height. The forward landing resulted in decreased changes in vertical velocity and a more forward body posture with upper extremity support at the end of the landing compared to the squat landing, which may provide advantages in maintaining posture stability and preparing forward movements. Fourth, the roll landing is recommended when individuals have to jump over a distance from the takeoff to the landing locations to minimize changes in horizontal velocity when the body is decelerating in the vertical direction. However, the roll landing involves the most complicated techniques and may require extensive training. Finally, participants had extensive training in Parkour, demonstrating skilled landing techniques and large joint range of motion. In addition, Grospretre and Lepers (2015) showed that Parkour practitioners demonstrated greater jump performance and eccentric knee strength compared to gymnasts, allowing them to better resist eccentric loading. Therefore, both landing techniques and physical characteristics, which 
may require long-term training, are important for performing effective landings from high heights.

Several limitations existed in the current study. Only three females participated, limiting the statistical power to detect potential sex differences. While visual inspection of the data suggests similar responses to landing heights and landing techniques in men and women, future studies need to include more female participants. Participants only performed each landing condition once, and the landing height was not randomized, in order to minimize injury risk. Trial-trial reliability could not be quantified. Only kinematic data were quantified. Assessing kinetic variables will provide important information for understanding movement strategies and injury risk (Zhang et al., 2000). The current video-based motion-capture method required researchers to manually digitize the locations of body landmarks. To minimize errors, three camcorders were used to ensure the visibility of body landmarks throughout the landing phase. In addition, researchers went through standard training of consistently locating body landmarks using an anatomical skeleton model. Compared to a marker-based motion capture method, the current method likely increased the errors introduced by researchers when the locations of certain landmarks were difficult to determine, but it might decrease the errors associated with participants' skin motion and the interference of markers on participants' movement patterns. The current video-based motion-capture method had a sampling frequency of $60 \mathrm{~Hz}$, lower than the frequencies in many previous studies (Dai et al., 2015a; McNitt-Gray, 1993). Although previous studies have supported the reliability of position and velocity data captured using the current method (Beardt et al., 2018; Dai et al., 2015b), good reliability does not mean the results are as valid as findings with higher frequencies. First, as the event of initial contact was visually identified, the error of the timing of this event ranged between 0 and $60^{\text {th }}$ of a second. Increasing the sampling frequency could improve the accuracy of the identification of initial contact and subsequently the calculation of all dependent variables. Second, the low sampling frequencies constrained the time intervals to quantify changes in velocities, because the error of timing of initial contact would have a greater effect on changes in velocities when the time interval was shorter. The low sampling frequency was one reason for calculating changes in velocities during the first $100 \mathrm{~ms}$ of landing compared to $50 \mathrm{~ms}$ or smaller time intervals. Also, if a time interval of $50 \mathrm{~ms}$ was used, it would only use three frames of data, which could be more sensitive to noise and errors. Third, a low sampling frequency also limited the ability to produce reliable acceleration. Based on the Newton's second law, the forces experienced by the body were directly related to its acceleration. Peak impact GRFs are important loading factors for the anterior cruciate ligament during landing, and peak impact GRFs could occur much earlier than 100 ms (Dai et al., 2015a). Ideally, peak acceleration and the timing of peak acceleration would be calculated and compared among the landing conditions. However, significant noise was observed for the acceleration of the whole-body COM calculated as the derivative of velocities. Vertical acceleration of the whole-body COM two frames prior to initial contact was $-9.4 \pm 3.2 \mathrm{~m} / \mathrm{s}^{2}$ for different landing conditions compared to a theoretical value of -9.8 $\mathrm{m} / \mathrm{s}^{2}$. This relatively large standard deviation did not allow us to make reliable comparisons among landing conditions for acceleration data. The changes of velocities during the first $100 \mathrm{~ms}$ of the landing were associated with the average forces applied to the participant, but should not be interpreted as the peak forces or peak loading experienced by the participant. Motion capture methods with higher sampling frequencies may be needed to obtain valid acceleration data. In addition, the acceleration experienced by individual segments such as the tibia can be much greater than the whole-body COM (Zhang et al., 2008). Measurements of acceleration of individual segments typically require accelerometers and should be considered in future studies.

In conclusion, the stiff landing demonstrated the shortest landing time and greatest changes in vertical velocity during the early landing, while the roll landing resulted in longest landing time and least changes in vertical and horizontal velocities during the early landing. The forward landing resulted in a more forward body posture at the end of the landing compared to the squat landing. The roll landing allowed individuals to decrease the downward and forward velocities over a longer time, which was 
likely to decrease the peak loading imposed on the lower extremities. The findings may provide information for developing landing training strategies for injury prevention and performance training.

\section{Acknowledgements}

The authors would like to thank Amos Rendao and Ryan Ford at the APEX Movement for their help with data collection. This study was supported by a grant from the National Institute of General Medical Sciences (5 U54 GM104944) from the National Institutes of Health. Jacob Layer's graduate assistantship was provided by the Wyoming INBRE, which was supported by a grant from the National Institute of General Medical Sciences (P20GM103432) from the National Institutes of Health.

\section{References}

Abdel-Aziz YI, Karara HM. Direct linear transformation from comparator coordinates into object space coordinates in close-range photogrammetry. Proceedings of the symposium on close-range photogrammetry. January 26-29, 1971. Urbana, IL, 1-18; 1971

Beardt BS, McCollum MR, Hinshaw TJ, Layer JS, Wilson MA, Zhu Q, Dai B. Lower extremity kinematics differed between a controlled drop-jump and volleyball-takeoffs. J Appl Biomech, 2018; 34: 327-335

Benjamini Y, Hochberg Y. Controlling the false discovery rate: A practical and powerful approach to multiple testing. J R Stat Soc Series B, 1995; 57: 289-300

Bennett HJ, Shen G, Weinhandl JT, Zhang S. Validation of the greater trochanter method with radiographic measurements of frontal plane hip joint centers and knee mechanical axis angles and two other hip joint center methods. J Biomech, 2016; 49: 3047-3051

Cruz A, Bell D, McGrath M, Blackburn T, Padua D, Herman D. The effects of three jump landing tasks on kinetic and kinematic measures: Implications for ACL injury research. Res Sports Med, 2013; 21: 330342

Dai B, Garrett WE, Gross MT, Padua DA, Queen RM, Yu B. The effects of 2 landing techniques on knee kinematics, kinetics, and performance during stop-jump and side-cutting tasks. Am J Sports Med, 2015a; 43: 466-474

Dai B, Garrett WE, Gross MT, Padua DA, Queen RM, Yu B. The effect of performance demands on lower extremity biomechanics during landing and cutting tasks. J Sport Health Sci, 2019; 8: 228-234

Dai B, Mao D, Garrett WE, Yu B. Anterior cruciate ligament injuries in soccer: Loading mechanisms, risk factors, and prevention programs. J Sport Health Sci, 2014; 3: 299-306

Dai B, Mao M, Garrett WE, Yu B. Biomechanical characteristics of an anterior cruciate ligament injury in javelin throwing. J Sport Health Sci, 2015b; 4: 333-340

de Leva P. Adjustments to zatsiorsky-seluyanov's segment inertia parameters. J Biomech, 1996; 29: 1223-1230

Devita P, Skelly WA. Effect of landing stiffness on joint kinetics and energetics in the lower extremity. Med Sci Sports Exerc, 1992; 24: 108-115

Dufek JS, Bates BT. The evaluation and prediction of impact forces during landings. Med Sci Sports Exerc, 1990; 22: 370-377

Ekeland A. Injuries in military parachuting: A prospective study of 4499 jumps. Injury, 1997; 28: 219-222

Grospretre S, Lepers R. Performance characteristics of parkour practitioners: Who are the traceurs? Eur J Sport Sci, 2015; 1-10

Hay JG. The biomechanics of sports techniques. Englewood Cliffs, NJ: Prentice-Hall. 1993: 138-143

Koga H, Nakamae A, Shima Y, Iwasa J, Myklebust G, Engebretsen L, Bahr R, Krosshaug T. Mechanisms for noncontact anterior cruciate ligament injuries: Knee joint kinematics in 10 injury situations from female team handball and basketball. Am J Sports Med, 2010; 38: 2218-2225 
Krosshaug T, Nakamae A, Boden BP, Engebretsen L, Smith G, Slauterbeck JR, Hewett TE, Bahr R. Mechanisms of anterior cruciate ligament injury in basketball: Video analysis of 39 cases. Am J Sports Med, 2007; 35: 359-367

Leigh S, Gross MT, Li L, Yu B. The relationship between discus throwing performance and combinations of selected technical parameters. Sports Biomech, 2008; 7: 173-193

Lin CF, Gross M, Ji C, Padua D, Weinhold P, Garrett WE, Yu B. A stochastic biomechanical model for risk and risk factors of non-contact anterior cruciate ligament injuries. J Biomech, 2009; 42: 418-423

McNitt-Gray JL. Kinetics of the lower extremities during drop landings from three heights. J Biomech, 1993; 26: 1037-1046

Owens BD, Mountcastle SB, Dunn WR, DeBerardino TM, Taylor DC. Incidence of anterior cruciate ligament injury among active duty U.S. military servicemen and servicewomen. Mil Med, 2007; 172: 90-91

Puddle DL, Maulder PS. Ground reaction forces and loading rates associated with parkour and traditional drop landing techniques. J Sports Sci Med, 2013; 12: 122-129

Sell TC, Chu Y, Abt JP, Nagai T, Deluzio J, McGrail MA, Rowe RS, Lephart, SM. Minimal additional weight of combat equipment alters air assault soldiers' landing biomechanics. Mil Med, 2010; 175: 41-47

$\mathrm{Yu}$ B, Andrews JG. The relationship between free limb motions and performance in the triple jump. J Appl Biomech, 1998; 14: 223-237

Zhang S, Derrick TR, Evans W, Yu YJ. Shock and impact reduction in moderate and strenuous landing activities. Sports Biomech, 2008; 7: 296-309

Zhang SN, Bates BT, Dufek JS. Contributions of lower extremity joints to energy dissipation during landings. Med Sci Sports Exerc, 2000; 32: 812-819

\section{Corresponding author:}

\section{Boyi Dai, PhD}

Division of Kinesiology and Health

University of Wyoming

Laramie, WY USA 82071

Phone: 1-3077665423; Fax: 1-3077664098;

Email: bdai@uwyo.edu 